\title{
CIpXP Protease Regulates the Type III Secretion System of Dickeya dadantii 3937 and Is Essential for the Bacterial Virulence
}

\author{
Yan Li, ${ }^{1,2}$ Akihiro Yamazaki, ${ }^{2}$ Lifang Zou, ${ }^{2}$ Eulandria Biddle, ${ }^{2}$ Quan Zeng, ${ }^{2}$ Yongjun Wang, ${ }^{3}$ Haiping Lin, ${ }^{3}$ \\ Qi Wang, ${ }^{1}$ and Ching-Hong Yang ${ }^{2}$ \\ ${ }^{1}$ Department of Plant Pathology, College of Agronomy \& Biotechnology, China Agricultural University, Beijing 100193, China; \\ ${ }^{2}$ Department of Biological Sciences, University of Wisconsin-Milwaukee 53211, U.S.A.; ${ }^{3}$ College of Forestry and \\ Biotechnology, Zhejiang Forestry University, Lin'an 311300, China
}

Submitted 16 December 2009. Accepted 24 March 2010.

\begin{abstract}
The type III secretion system (T3SS) is considered one of the major virulence factors in many bacterial pathogens. This report demonstrates that $\operatorname{RssB}$, ClpXP, and $\mathrm{RpoS}$ play a role in T3SS regulation of Dickeya dadantii 3937. ClpP is a serinetype protease which associates with the ClpX chaperone to form a functional Clp proteolytic complex for degradation of proteins. With the assistance of recognition factor RssB, ClpXP degrades the RpoS sigma factor. RpoS positively regulates the expression of the $\operatorname{rsm} A$ gene encoding an RNAbinding regulatory protein. By interacting with the $h r p L$ mRNA, RsmA reduces HrpL production and downregulates the T3SS genes in the HrpL regulon. In addition, ClpXP, RssB, and RpoS affect pectinolytic enzyme production in $D$. dadantii 3937, probably through RsmA. The ClpXP and RssB proteins are essential for bacterial virulence.
\end{abstract}

Dickeya dadantii 3937 (formerly named Erwinia chrysanthemi), a member of the Enterobacteriaceae family, is an opportunistic necrotrophic pathogen that causes soft rot, wilts, and blight diseases on a wide range of plants, including ornamental plants and economically important vegetable crops $(\mathrm{Ma}$ et al. 2007). Several virulence determinants have been discovered in $D$. dadantii. These include the well-studied extracellular enzymes such as pectate lyases (Pel), polygalacturonases (Peh), cellulases, and proteases (Prt), with Pel as the major virulence determinant among these isozymes (Collmer and Keen 1986; Roy et al. 1999; Herron et al. 2000; Kazemi-Pour et al. 2004). Siderophore-dependent iron uptake systems and motility of the bacterial strains are also required for pathogenicity (Franza et al. 1999; Antunez-Lamas et al. 2009). In addition to the above-named virulence factors, the type III protein secretion system (T3SS) is reported to play an important role in the initial infection of the bacterium in plant hosts (Bauer et al. 1994; Yang et al. 2002). In gram-negative pathogens, the T3SS translocates effector proteins directly into the host cell cytosol to cause disease symptoms (Hueck 1998; He et al. 2004; Mota et al. 2005).

In $D$. dadantii 3937 , at least two main regulatory pathways leading to the activation of the T3SS in the bacterium have

Corresponding authors: Ching-Hong Yang; Telephone: +1-414-229-6331; Fax: +1-414-229-3926; E-mail: chyang@uwm.edu; and Qi Wang; E-mail: wangqi@cau.edu.cn

* The $\boldsymbol{e}$-Xtra logo stands for "electronic extra" and indicates that one supplementary figure and one supplementary table are published online. been proposed (Fig. 1) (Yap et al. 2005; Tang et al. 2006; Yang et al. 2008a,b; Li et al. 2009). In the HrpX/Y-HrpS-HrpL regulatory pathway, the two-component system HrpX/HrpY activates $h r p S$, which encodes a $\sigma^{54}$-enhancer binding protein. HrpS interacts with the $\sigma^{54}(\mathrm{RpoN})$-containing RNA polymerase holoenzyme and initiates transcription of hrpL (Chatterjee et al. 2002; Yap et al. 2005; Tang et al. 2006). HrpL is a member of the extracytoplasmic function (ECF) family of alternative sigma factors and is the central regulator required for activation of many genes downstream of the T3SS regulatory cascade, such as $h r p A, d s p E$, and $h r p N$, which encode a structural protein of the T3SS pilus, a T3SS effector, and a T3SS harpin, respectively (Wei and Beer 1995; Chatterjee et al. 2002; Tang et al. 2006). In the Rsm-mediated regulatory pathway, RsmA (a small RNA-binding protein) can bind to the $h r p L$ mRNA to facilitate its degradation in Pectobacterium carotovorum (Chatterjee et al. 1995, 2002; Cui et al. 1995) (Fig. 1). Alternatively, an untranslated regulatory RNA molecule, $r \operatorname{smB}$, binds to RsmA and neutralizes its degradation effect on $h r p L$ mRNA (Liu et al. 1998; Chatterjee et al. 2002). Another global twocomponent system, GacS/A, upregulates expression of $r s m B$, which in turn, inactivates RsmA, resulting in elevated levels of hrpL mRNA (Tang et al. 2006; Yang et al. 2008b).

Proteolysis plays a critical role in maintaining cellular homeostasis by controlling the levels of various proteins, including global regulators involved in numerous biological functions, as well as misfolded and damaged proteins (Jenal and HenggeAronis 2003; Ehrmann and Clausen 2004). In bacteria, most intracellular proteolysis is performed by the energy-dependent proteases Clp and Lon (Gottesman 1996, 2003; Porankiewicz et al. 1999; Jenal and Hengge-Aronis 2003). Proteases are grouped into different categories according to an active site residue or metal involved in the catalytic activity, such as serine or threonine proteases, and metalloproteases (Ehrmann and Clausen 2004). ClpP, a serine-type protease, is highly conserved throughout prokaryotes and eukaryotes. ClpP typically interacts with the ATP-dependent AAA+ chaperones, ClpX or ClpA. These chaperones determine the substrate specificity and also unfold and translocate the substrate to the proteolytic chamber where proteins are degraded into short peptides (Dougan et al. 2002; Yu and Houry 2007).

In response to environmental signals, the $\mathrm{ClpP}$ protease performs important and diverse roles in bacteria by rapid regulation of cellular levels and activities of regulatory proteins or sigma factors. This system allows the cell to alter its pattern of gene expression to meet changing needs. For example, ClpXP 
protease regulates the activity of RpoE by degrading the cytoplasmic domain of the single-pass inner membrane protein RseA, which frees RpoE to interact with RNA polymerase (Ades et al. 1999). RpoE is an ECF family sigma factor involved in sensing cell envelope stress and plays a role in the virulence of some bacteria (Missiakas et al. 1997; Hayden and Ades 2008). In addition, the virulence-associated T3SS was reported to be indirectly regulated by $\mathrm{ClpXP}$ through regulation of sigma factor RpoS $\left(\sigma^{\mathrm{S}}\right)$, GrlR, GadE, and GadX in enterohemorrhagic Escherichia coli (Iyoda and Watanabe 2005; Tomoyasu et al. 2005). The RpoS protein is a master regulator for the general stress response in bacteria (Loewen et al. 1998;

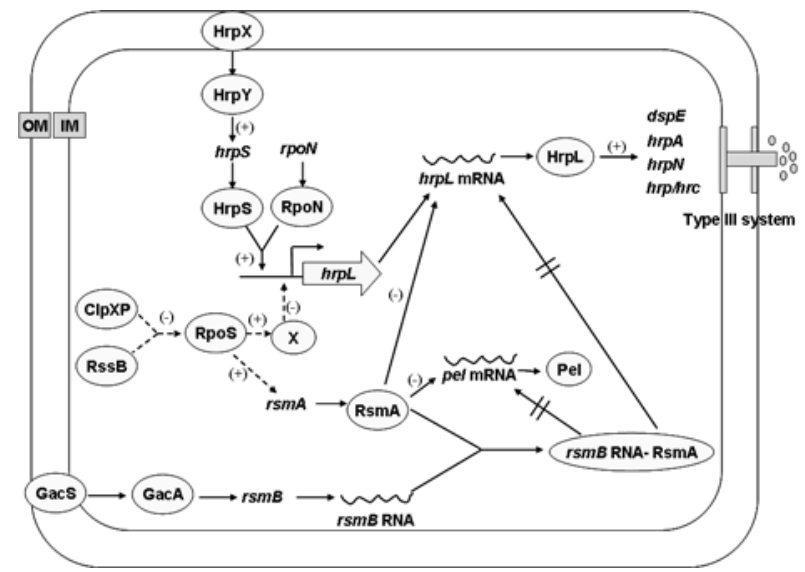

Fig. 1. Regulatory network controlling the Dickeya dadantii 3937 type III secretion system (T3SS) and the production of pectate lyases. The $D$. dadantii 3937 T3SS is regulated by the HrpX/HrpY-HrpS-HrpL and the $\mathrm{GacS} / \mathrm{GacA}$-rsmB-HrpL regulatory pathways. Dashed lines represent regulators identified in this article. ClpXP and RssB promote RpoS (an alternative sigma factor) degradation. RpoS negatively regulates $h r p L$ by downregulating the promoter activity of $h r p L$ and upregulating global repressor RsmA. RsmA can bind to and cause the degradation of the $h r p L$ mRNA and, in turn, downregulate genes in the HrpL regulon, such as hrpA, hrpN, and $d s p E$. RsmA also regulates the production of pectate lyases (Pel). Symbols: + designates positive regulation and - designates negative regulation.
Hengge-Aronis 2002; McMeechan et al. 2007; Sandercock and Page 2008), and is present at very low levels during exponential cell growth in media, largely due to its rapid degradation by ClpXP protease (Lange and Henggearonis 1994; Schweder et al. 1996). RpoS degradation by ClpXP takes place with the assistance of a recognition factor, RssB, which delivers RpoS to ClpXP for degradation (Zhou et al. 2001). In $P$. carotovorum, RpoS was reported to affect the extracellular enzyme levels, $h r p N_{E c c}$ expression, and virulence of the bacterium (Mukherjee et al. 1998).

Limited information is available about the regulatory effect of ClpXP on T3SS and the role of ClpXP in the virulence of phytobacteria. This study demonstrates that, with the help of RssB, ClpXP positively regulates T3SS through RpoS degradation. In addition to the regulation of T3SS, ClpXP protease, $\mathrm{RssB}$, and RpoS play a role in pectinolytic enzyme production and virulence of $D$. dadantii 3937.

\section{RESULTS AND DISCUSSION}

\section{Regulatory effect of ClpXP on T3SS.}

A $\operatorname{clpP}$ mutant of $D$. dadantii 3937 was obtained by insertion of the transposon miniHimar RB1, on the basis of an altered $h r p A$ expression in minimal medium (MM) (data not shown). In order to investigate the role of $c l p P$ in T3SS regulation, we constructed a $\operatorname{clp} P$ deletion mutant (Ech156) (Table 1) and evaluated the effects of this mutation on expression of the T3SS genes $h r p A, h r p N$, and $d s p E$. These genes are located downstream in the HrpL regulon of D. dadantii 3937. Employing a green fluorescent protein (GFP) reporter system, promoter activities of $h r p A, h r p N$, and $d s p E$ of Ech156 were measured by flow cytometry. Deletion of $c l p P$ resulted in reduced expression of $h r p A, h r p N$, and $d s p E$ compared with the wild type (Table 2). The promoter activity of $h r p A$ was restored to wildtype level when Ech156 was complemented with pMLclpP (Table 3). Because $h r p A, h r p N$, and $d s p E$ are all regulated by HrpL, hrpA was selected as the representative T3SS gene for the Northern blot and complementation assays. The effect of ClpP on hrpA expression was confirmed by Northern blot. Compared with the wild type, a smaller amount of $h r p A$

Table 1. Strains and plasmids used in this study

\begin{tabular}{|c|c|c|}
\hline Strains and plasmids & Characteristics $^{\mathbf{a}}$ & Reference or source \\
\hline \multicolumn{3}{|l|}{ Strains } \\
\hline \multicolumn{3}{|l|}{ Dickeya dadantii } \\
\hline 3937 & Wild type, Saintpaulia (African violet) isolate & N. Hugouvieux-Cotte-Pattat \\
\hline Ech 156 & $\Delta c l p P:: k a n, \mathrm{Km}^{\mathrm{r}}$ & This work \\
\hline Ech 157 & $\Delta c l p X:: k a n, \mathrm{Km}^{\mathrm{r}}$ & This work \\
\hline Ech 158 & $\Delta c l p X P:: k a n ; \mathrm{Km}^{\mathrm{r}}$ & This work \\
\hline Ech 159 & $\Delta r p o S:: k a n, \mathrm{Km}^{\mathrm{r}}$ & This work \\
\hline Ech 160 & $\Delta r s s B:: k a n, \mathrm{Km}^{\mathrm{r}}$ & This work \\
\hline Ech161 & $\Delta r p o E:: k a n, \mathrm{Km}^{\mathrm{r}}$ & This work \\
\hline \multicolumn{3}{|l|}{ Escherichia coli } \\
\hline E. coli $\mathrm{S} 17-1 \lambda$-pir & $\lambda$-pir Lysogen of S17-1, Sp ${ }^{r}$ & Victor de Lorenzo, Spain \\
\hline \multicolumn{3}{|l|}{ Plasmids } \\
\hline pPROBE-AT & Promoter-probe vector, $A p^{r}$ & Miller et al. 2001 \\
\hline phrpA & pProbe-AT derivative with PCR fragment containing $h r p A$ promoter region, $\mathrm{Ap}^{\mathrm{r}}$ & Yang et al. 2008b \\
\hline phrpN & pProbe-AT derivative with PCR fragment containing hrpN promoter region, Ap $^{\mathrm{r}}$ & Yang et al. 2007 \\
\hline pdspE & pProbe-AT derivative with PCR fragment containing $d s p E$ promoter region, $\mathrm{Ap}^{\mathrm{r}}$ & Peng et al. 2006 \\
\hline phrpS & pProbe-AT derivative with PCR fragment containing hrpS promoter region, Ap $^{\mathrm{r}}$ & Yang et al. 2007 \\
\hline phrpL & pProbe-AT derivative with PCR fragment containing hrpL promoter region, Ap ${ }^{\mathrm{r}}$ & Yang et al. 2007 \\
\hline pWM91 & Sucrose-based counter-selectable plasmid, $\mathrm{Ap}^{\mathrm{r}}$ & Metcalf et al. 1996 \\
\hline pML122/123 & RSF1010-derived expression and lac-fusion broad host-range vector, $\mathrm{Gm}^{\mathrm{r}}$ & Labes et al. 1990 \\
\hline pMLclpP & pML122 derivative with PCR fragment containing $c l p P, \mathrm{Gm}^{\mathrm{r}}$ & This work \\
\hline pMLclpXP & pML123 derivative with PCR fragment containing $\operatorname{clp} X P, \mathrm{Gm}^{\mathrm{r}}$ & This work \\
\hline pMLrpoS & pML123 derivative with PCR fragment containing rpos, $\mathrm{Gm}^{\mathrm{r}}$ & This work \\
\hline pMLrssB & pML123 derivative with PCR fragment containing $r s s B, \mathrm{Gm}^{\mathrm{r}}$ & This work \\
\hline pKD4 & Template plasmid for kanamycin cassette, $\mathrm{Km}^{\mathrm{r}}$ & Datsenko and Wanner 2000 \\
\hline
\end{tabular}

\footnotetext{
${ }^{a} \mathrm{Ap}^{\mathrm{r}}, \mathrm{Km}^{\mathrm{r}}, \mathrm{Sp}^{\mathrm{r}}$, and $\mathrm{Gm}^{\mathrm{r}}$ indicate resistance to ampicillin, kanamycin, spectinomycin, and gentamicin, respectively. $\mathrm{PCR}=$ polymerase chain reaction.
} 
mRNA was observed in Ech156 (Fig. 2A). In addition, the hrpA mRNA level in Ech156 was restored to wild-type level upon complementation of Ech156 with pMLclpP (Fig. 2A).

ClpX is a Clp ATPase, which associates with ClpP to form a functional proteolytic complex for the degradation of proteins (Ortega et al. 2004; Butler et al. 2006). clpX and $\operatorname{clpP}$ of $D$. dadantii 3937 are arranged in an operon. To investigate whether an interaction between ClpX and ClpP is necessary for regulation of the T3SS in $D$. dadantii, a $\operatorname{clp} X$ deletion mutant (Ech157) and a clpX, clpP double-deletion mutant (Ech158) were constructed. Compared with D. dadantii 3937, hrpA promoter activity was reduced by factors of 5 to 13 in Ech157 and Ech158 using the GFP reporter system (Table 2). When pMLclpXP containing the entire $\operatorname{clpXP}$ operon was provided in trans, the $h r p A$ expression level was restored in both mutants (Table 3). These results indicate that the ATP-dependent proteolytic complex ClpXP acts as a positive regulator of T3SS genes.

\section{ClpXP regulates $h r p L$}

at transcriptional and post-transcriptional levels.

Given that ClpXP positively regulates $h r p A$ and $h r p N$, we also investigated the regulatory effect of ClpXP on key compo-

Table 2. Expression of type III secretion system genes in Dickeya dadantii 3937 (3937), clpP mutant (Ech156), clpX mutant (Ech157), clpXP mutant (Ech158), rpoS mutant (Ech159), and rssB mutant (Ech160) ${ }^{\mathrm{a}}$

\begin{tabular}{|c|c|c|}
\hline \multirow[b]{2}{*}{ Strain } & \multicolumn{2}{|c|}{ Average MFI \pm standard deviation at } \\
\hline & $12 \mathrm{~h}$ & $24 \mathrm{~h}$ \\
\hline 3937 (phrpA) & $55.3 \pm 6.1$ & $77.3 \pm 10.4$ \\
\hline Ech156 (phrpA) & $8.7 \pm 0.5^{*}$ & $7.0 \pm 0.3^{*}$ \\
\hline 3937 (phrpN) & $32.7 \pm 10.7$ & $56.4 \pm 9.8$ \\
\hline Ech156 (phrpN) & $4.6 \pm 0.4^{*}$ & $3.6 \pm 0.1^{*}$ \\
\hline 3937 (pdspE) & $20.1 \pm 0.7$ & $30.1 \pm 0.6$ \\
\hline Ech156 (pdspE) & $4.8 \pm 0.2 *$ & $4.0 \pm 0.3^{*}$ \\
\hline 3937 (phrpL) & $9.0 \pm 0.3$ & $11.0 \pm 0.4$ \\
\hline Ech156 (phrpL) & $6.5 \pm 0.5^{*}$ & $5.6 \pm 0.2 *$ \\
\hline 3937 (phrpA) & $52.6 \pm 9.3$ & $88.9 \pm 13.5$ \\
\hline Ech157 (phrpA) & $11.4 \pm 0.2 *$ & $11.4 \pm 0.4^{*}$ \\
\hline $3937(\mathrm{phrpN})$ & $42.3 \pm 1.8$ & $73.1 \pm 3.2$ \\
\hline Ech157 (phrpN) & $6.3 \pm 0.3^{*}$ & $5.9 \pm 0.2^{*}$ \\
\hline 3937 (phrpL) & $13.1 \pm 0.1$ & $19.1 \pm 0.6$ \\
\hline Ech157 (phrpL) & $9.4 \pm 0.8^{*}$ & $9.7 \pm 0.8^{*}$ \\
\hline 3937 (phrpA) & $57.1 \pm 4.6$ & $72.1 \pm 6.9$ \\
\hline Ech158 (phrpA) & $7.4 \pm 0.4^{*}$ & $5.2 \pm 0.3^{*}$ \\
\hline 3937 (phrpN) & $54.2 \pm 4.0$ & $71.3 \pm 5.1$ \\
\hline Ech158 (phrpN) & $4.0 \pm 0.4^{*}$ & $3.0 \pm 0.3 *$ \\
\hline 3937 (phrpL) & $14.9 \pm 0.6$ & $20.8 \pm 1.1$ \\
\hline Ech158 (phrpL) & $5.1 \pm 0.6^{*}$ & $6.7 \pm 0.5^{*}$ \\
\hline 3937 (phrpA) & $55.3 \pm 6.1$ & $77.3 \pm 10.4$ \\
\hline Ech159 (phrpA) & $116.6 \pm 15.0^{*}$ & $189.6 \pm 6.9 *$ \\
\hline 3937 (phrpN) & $32.7 \pm 10.7$ & $56.4 \pm 9.8$ \\
\hline Ech159 (phrpN) & $88.6 \pm 4.3^{*}$ & $180.1 \pm 7.5^{*}$ \\
\hline 3937 (pdspE) & $20.1 \pm 0.7$ & $30.1 \pm 0.6$ \\
\hline Ech159 (pdspE) & $33.6 \pm 2.5^{*}$ & $87.5 \pm 2.2^{*}$ \\
\hline 3937 (phrpL) & $9.0 \pm 0.3$ & $11.0 \pm 0.4$ \\
\hline Ech159 (phrpL) & $22.8 \pm 3.6^{*}$ & $32.4 \pm 0.4^{*}$ \\
\hline 3937 (phrpA) & $52.6 \pm 9.3$ & $88.9 \pm 13.5$ \\
\hline Ech160 (phrpA) & $9.8 \pm 0.3 *$ & $11.2 \pm 0.1^{*}$ \\
\hline 3937 (phrpN) & $42.3 \pm 1.8$ & $73.1 \pm 3.2$ \\
\hline Ech160 (phrpN) & $5.2 \pm 0.5^{*}$ & $5.4 \pm 0.3^{*}$ \\
\hline 3937 (phrpL) & $13.1 \pm 0.1$ & $19.1 \pm 0.6$ \\
\hline Ech160 (phrpL) & $10.2 \pm 0.2 *$ & $11.5 \pm 0.2 *$ \\
\hline 3937 (pPROBE-AT) & $2.9 \pm 0.0$ & $4.9 \pm 0.1$ \\
\hline
\end{tabular}

a Promoter activities at 12 and $24 \mathrm{~h}$ of bacterial growth were determined. Green fluorescent protein (GFP) mean fluorescence intensity (MFI) was determined for gated populations of bacterial cells by flow cytometry. Values are representative of two or three experiments, and three replicates were used for each experiment. Asterisks indicate statistically significant differences in GFP MFI between the wild type (3937) and mutants $(P<0.01$, Student's $t$ test $)$. nents of the T3SS regulatory pathways. Because HrpL is the main regulator required for activation of genes downstream of the regulatory pathways, such as $h r p A, h r p N$, and $d s p E$ (Wei and Beer 1995; Chatterjee et al. 2002; Tang et al. 2006), we examined the effect of the $\operatorname{clpP}$ mutation (Ech156) on $h r p L$ by quantitative reverse-transcription polymerase chain reaction (qRT-PCR). In Ech156, the amount of $h r p L$ mRNA was only 0.23 -fold that of the wild-type strain (Fig. 3A). In addition, a decrease in $h r p L$ promoter activity was observed in Ech156, Ech157, and Ech158 compared with the wild type at 12 and 24 $\mathrm{h}$ using the GFP reporter system in combination with flow cytometry (Table 2). These results suggest that ClpXP positively regulates $h r p L$ expression.

Table 3. Expression of type III secretion system gene hrpA in Dickeya dadantii 3937 (3937), clpP mutant (Ech156), clpX mutant (Ech157), clpXP mutant (Ech158), rpoS mutant (Ech159), and rssB mutant (Ech160) containing pML122/123, or complementation plasmid ${ }^{\mathrm{a}}$

\begin{tabular}{lcc}
\hline & \multicolumn{2}{c}{ Average MFI \pm standard deviation at } \\
\cline { 2 - 3 } Stain $^{\mathbf{b}}$ & $\mathbf{1 2} \mathbf{~ h}$ & $\mathbf{2 4 ~ h}$ \\
\hline 3937 (pML122) & $21.4 \pm 1.4$ & $39.0 \pm 3.6$ \\
Ech156 (pML122) & $9.0 \pm 0.7^{*}$ & $7.2 \pm 0.3^{*}$ \\
Ech156 (pMLclpP) & $23.8 \pm 4.3$ & $39.1 \pm 7.2$ \\
3937 (pML123) & $43.3 \pm 1.5$ & $47.8 \pm 2.2$ \\
Ech157 (pML123) & $18.2 \pm 0.2^{*}$ & $14.3 \pm 0.5^{*}$ \\
Ech157 (pMLclpXP) & $51.7 \pm 5.4$ & $84.8 \pm 7.9^{*}$ \\
3937 (pML123) & $43.3 \pm 1.5$ & $47.8 \pm 2.2$ \\
Ech158 (pML123) & $12.0 \pm 0.6^{*}$ & $8.3 \pm 0.3^{*}$ \\
Ech158 (pMLclpXP) & $45.5 \pm 4.2$ & $113.6 \pm 5.5^{*}$ \\
3937 (pML123) & $27.2 \pm 4.7$ & $62.2 \pm 7.8$ \\
Ech159 (pML123) & $56.1 \pm 1.0^{*}$ & $121.4 \pm 1.5^{*}$ \\
Ech159 (pMLrpoS) & $9.9 \pm 0.4^{*}$ & $10.1 \pm 0.3^{*}$ \\
3937 (pMLrpoS) & $10.7 \pm 0.5^{*}$ & $9.7 \pm 0.2^{*}$ \\
3937 (pML123) & $43.3 \pm 1.5$ & $47.8 \pm 2.2$ \\
Ech160 (pML123) & $15.9 \pm 1.3^{*}$ & $14.5 \pm 0.4^{*}$ \\
Ech160 (pMLrssB) & $92.0 \pm 0.4^{*}$ & $106.2 \pm 4.5^{*}$ \\
\hline
\end{tabular}

${ }^{\text {a }}$ Promoter activity of $h r p A$ was compared at 12 and $24 \mathrm{~h}$ of bacterial growth in strains containing empty vector pML122/123 or complementation plasmid. Green fluorescent protein (GFP) mean fluorescence intensity (MFI) was determined for gated populations of bacterial cells by flow cytometry. Values are representative of two experiments, and three replicates were used for each experiment. Asterisks indicate statistically significant differences in GFP MFI between the wild-type strain (3937) with empty vector and mutants with empty vector or complementation plasmid ( $P<0.01$, Student's $t$ test).

${ }^{b}$ Stains carrying two plasmids (one was GFP reporter phrpA and the other was pML122/123 or their derivatives) were used in this study.

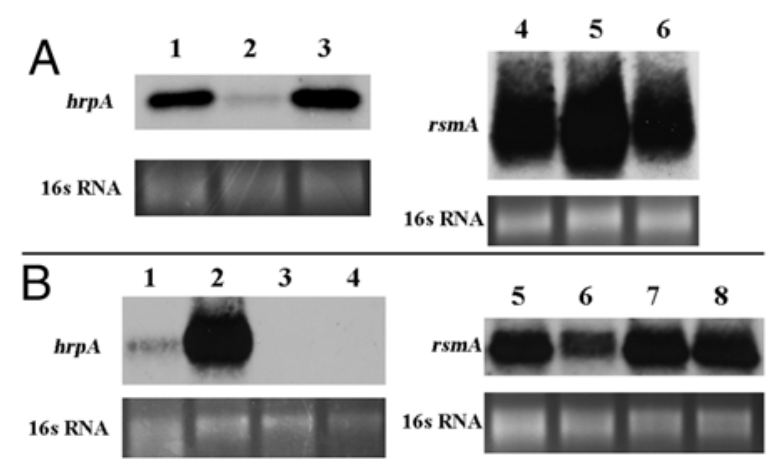

Fig. 2. Northern blot analysis of $h r p A$ and $r s m A$ mRNA levels. A, hrpA and rsmA mRNA levels in lanes 1 and 4, Dickeya dadantii 3937 with pML122; lanes 2 and 5, Ech156 (clpP mutant) with pML122; and lanes 3 and 6 , Ech156 with complementation plasmid pMLclpP. B, hrpA and rsmA mRNA levels in lanes 1 and 5, D. dadantii 3937 with pML123; lanes 2 and 6, Ech159 (rpoS mutant) with pML123; lanes 3 and 7, Ech159 with complementation plasmid pMLrpoS; and lanes 4 and 8, D. dadantii 3937 with pMLrpoS. Cells were cultured in minimal medium for $12 \mathrm{~h}$ before the RNA isolation. 16S rRNA was used as an internal control. 
The T3SS of $D$. dadantii 3937 is controlled by two major regulatory pathways, HrpX/Y-HrpS and GacS/A-rsmB-RsmA (Yap et al. 2005; Tang et al. 2006; Yang et al. 2008a,b; Li et al. 2009). To determine the T3SS regulatory pathway through which ClpXP controls hrpL, the expression of $h r p S$ was examined in the wild type and the $\operatorname{clpP}$ mutant (Ech156) by qRTPCR. The hrpS mRNA level in Ech156 was comparable with that in the wild-type strain (Fig. 3A), indicating that the regulatory effect on $h r p L$ by ClpXP is probably not through the HrpX/Y-HrpS pathway.

We also evaluated the effect of ClpXP on T3SS gene expression through the RsmA-rsmB-dependent regulatory pathway. The RNA levels of $r s m A$ and $r s m B$ were examined in the wild type and Ech156. Northern blot analysis revealed a considerable increase in $r s m A$ mRNA in Ech156 at $12 \mathrm{~h}$ of growth in MM (Fig. 2A) but the level of $r s m B$ RNA in Ech156 was indistinguishable from that detected in the wild type (Supplementary Fig. 1). Complementation of Ech156 with pMLclpP restored $r s m A$ mRNA roughly to the wild-type level (Fig. 2A). In $P$. carotovorum, RsmA negatively regulates $h r p L$ at the posttranscriptional level to ultimately regulate genes in the HrpL regulon (Cui et al. 1995; Chatterjee et al. 2002). Our results indicate that ClpXP functions upstream in the post-
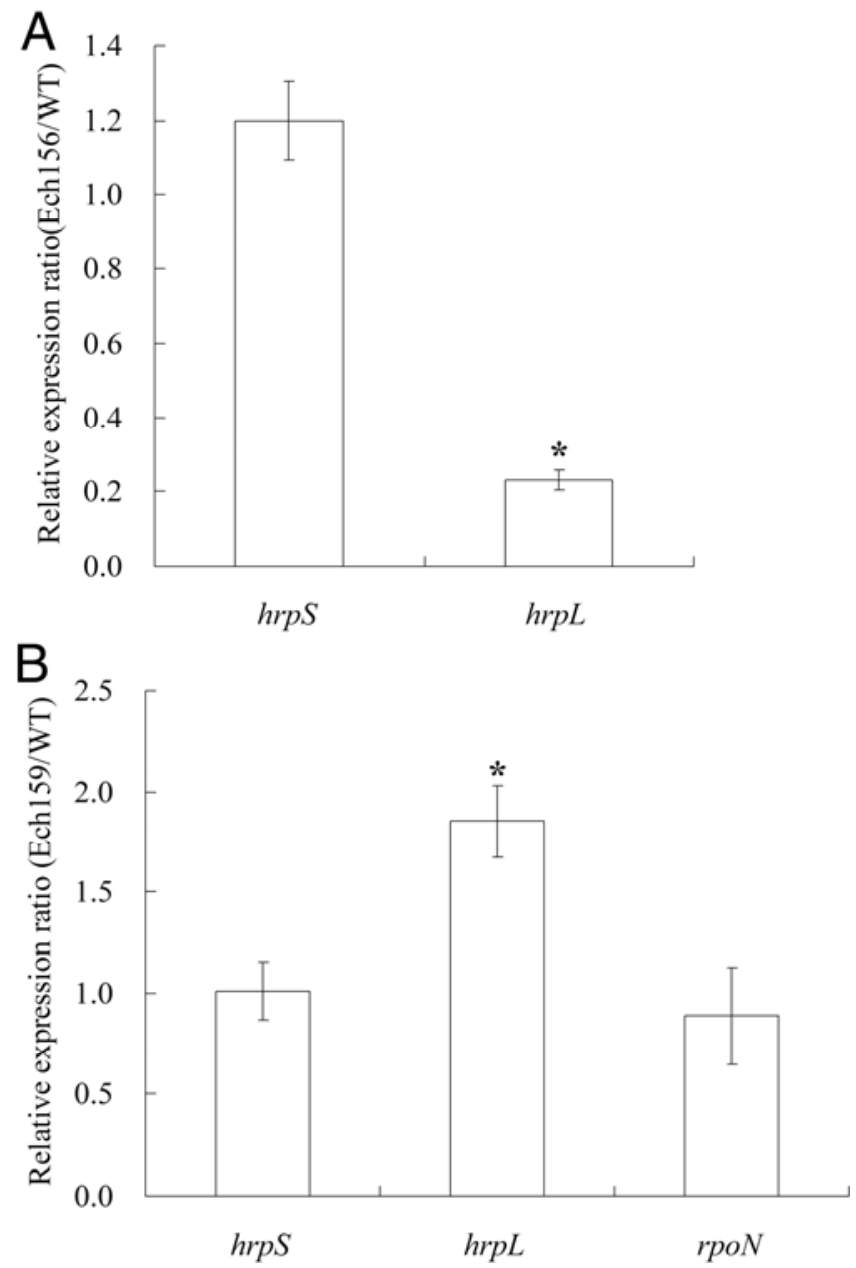

Fig. 3. Relative mRNA levels of type III secretion system (T3SS) genes in mutants and Dickeya dadantii 3937 (WT) as determined by quantitative reverse-transcription polymerase chain reaction. A, Relative mRNA level of $h r p S$ and $h r p L$ in WT and the $c l p P$ mutant (Ech156). B, Relative mRNA levels of $h r p S$, $h r p L$, and rpoN in WT and the $r p o S$ mutant (Ech159). rplU was used as an endogenous control for data analysis. Asterisks indicate statistically significant differences in mRNA level of the mutants compared with WT $(P<0.05$, Student's $t$ test $)$. transcriptional regulatory pathway and positively regulates $h r p L$ and genes in the HrpL regulon through downregulation of $r s m A$ expression (Fig. 1).

In summary, a deletion in $\operatorname{clpP}$ (Ech156) led to an increase in the $r s m A$ mRNA level and a decrease in the hrpL mRNA level as well as a decrease in $h r p L$ promoter activity (Figs. 2 and 3; Table 2). These results suggest that ClpXP positively regulates $h r p L$ at both transcriptional and post-transcriptional levels.

\section{Effect of ClpXP on T3SS gene expression is not channeled via RpoE.}

The activity of the stress responsive sigma factor RpoE is controlled by the level of anti-sigma factor RseA. The ClpXP protease degrades the cytoplasmic domain of the RseA protein in response to stress, which leads to increased levels of free RpoE that can interact with RNA polymerase (Ades et al. 1999). Our results show that ClpXP negatively regulates the level of $r s m A$ mRNA but the regulatory mechanism is unclear. To investigate the possibility that ClpXP may regulate the expression of T3SS genes in an RpoE-dependent manner, an rpoE mutant (Ech161) was constructed. Using the GFP promoter probe, promoter activities of $h r p S, h r p L$, and $h r p N$ were measured and found to be similar at 12 and $24 \mathrm{~h}$ in the wildtype strain and rpoE mutant grown in MM (Table 4). These results demonstrate that the effect of ClpXP on T3SS gene expression is not channeled via RpoE.

\section{RpoS regulates T3SS by mediating $r \operatorname{sm} A$ expression.}

In enterohemorrhagic E. coli, ClpXP was reported to control T3SS through degradation of RpoS (Iyoda and Watanabe 2005; Tomoyasu et al. 2005). In addition, RpoS was reported to regulate $r s m A$ and harpin production in $P$. carotovorum (Mukherjee et al. 1998). Based on this information, we hypothesized that i) RpoS may regulate T3SS in $D$. dadantii 3937 and ii) the ClpXP proteolytic complex may also regulate T3SS through degradation of RpoS in this bacterium. To test the regulatory effect of RpoS on T3SS, an rpoS null mutant (Ech159) was constructed and the promoter activities of hrp genes were examined by flow cytometry using the GFP promoter probe. Compared with $D$. dadantii 3937, the expression levels of $h r p A, h r p N$, and $d s p E$ were increased twoto threefold in Ech159 (Table 2). The mRNA level of hrpA in D. dadantii 3937 and Ech159 was also examined by Northern blot. Compared with the wild-type strain, an increase in hrpA mRNA was observed in Ech159 (Fig. 2B). Moreover, hrpA expression was dramatically reduced by introduction of pMLrpoS into the rpoS mutant or the wild type compared with either strain containing only the vector (Table 3; Fig. 2B). These results demonstrate that RpoS negatively regu-

Table 4. Promoter activities of type III secretion system genes in Dickeya dadantii 3937 (3937) and rpoE mutant (Ech161)a

\begin{tabular}{lcc}
\hline & \multicolumn{2}{c}{ Average MFI \pm standard deviation at } \\
\cline { 2 - 3 } Stain & $\mathbf{1 2 ~ h}$ & $\mathbf{2 4} \mathbf{~ h}$ \\
\hline 3937(phrpS) & $50.9 \pm 2.1$ & $73.0 \pm 1.6$ \\
Ech161(phrpS) & $49.4 \pm 2.4$ & $76.7 \pm 2.0$ \\
3937(phrpL) & $7.7 \pm 0.4$ & $12.6 \pm 0.4$ \\
Ech161(phrpL) & $8.5 \pm 0.2$ & $10.9 \pm 0.7$ \\
3937(phrpN) & $45.0 \pm 6.7$ & $65.8 \pm 6.8$ \\
Ech161(phrpN) & $37.8 \pm 1.2$ & $68.4 \pm 4.7$ \\
3937(pPROBE-AT) & $3.3 \pm 0.4$ & $4.3 \pm 0.6$ \\
\hline
\end{tabular}

${ }^{a}$ Promoter activities at 12 and $24 \mathrm{~h}$ of bacterial growth were determined. Green fluorescent protein (GFP) mean fluorescence intensity (MFI) was determined for gated populations of bacterial cells by flow cytometry. Values are representative of three experiments, and three replicates were used for each experiment. 
lates expression of genes encoding T3SS effectors and structural components.

In $P$. carotovorum, RpoN $\left(\sigma^{54}\right)$ is required for the transcription of hrpL (Chatterjee et al. 2002). We speculated that RpoS may regulate the expression of rpoN and, in turn, the RpoN protein regulates $h r p L$ expression. To test this hypothesis, the rpoN mRNA level was measured in the wild type and the rpoS mutant by qRT-PCR. We found that the rpoN mRNA level in Ech159 was similar to that in the wild type (Fig. 3B), suggesting that RpoS does not regulate the expression of rpoN.

To elucidate the regulatory mechanism of RpoS on T3SS, the expression of key T3SS regulatory genes hrpS, rsmA, $r s m B$, and $h r p L$ were examined in the wild type and Ech159. Northern blot analysis revealed a considerable decrease in rsmA mRNA in cell extracts of Ech159 grown in MM for $12 \mathrm{~h}$ (Fig. 2B) but no difference was observed in the level of $r s m B$ RNA. Complementation of Ech159 with pMLrpoS restored rsmA mRNA almost to the wild-type level (Fig. 2B). As observed by qRT-PCR, the hrpS mRNA level in Ech159 was comparable with that in the wild-type strain but the hrpL mRNA level was significantly higher in Ech159 (Fig. 3B). Finally, the promoter activity of $h r p L$ was examined by GFP promoter probe. After growth in MM, the promoter activity of $h r p L$ was increased two- to threefold in Ech159 compared with the wild type at 12 and $24 \mathrm{~h}$ (Table 2).

In summary, the rpoS mutant (Ech159) exhibited a decrease in $r s m A$ mRNA and an increase in the levels of $h r p L$ mRNA and hrpL promoter activity (Figs. 2 and 3; Table 2). These results indicate that RpoS regulates the expression of genes encoding T3SS effectors and structural components by mediating $r s m A$ expression, which results in a reduction of $h r p L$ mRNA stability. RpoS also negatively regulates $h r p L$ at the transcriptional level. At this stage, how RpoS affects the promoter activity of $h r p L$ is unclear. It is possible that RpoS upregulates an unidentified transcriptional repressor of $h r p L$ which further controls the expression of hrpL (Fig. 1).

\section{ClpXP regulates $r s m A$}

\section{through RssB-mediated RpoS degradation.}

In several gram-negative bacteria, the $\operatorname{RpoS}\left(\sigma^{S}\right)$ protein is a master regulator of the general stress response (Loewen et al. 1998; McMeechan et al. 2007; Sandercock and Page 2008). With the assistance of the recognition factor RssB, RpoS is rapidly degraded by ClpXP protease during exponential cell growth in E. coli (Lange and Henggearonis 1994; Schweder et al. 1996; Zhou et al. 2001). To verify the regulatory effect of RssB on T3SS genes of $D$. dadantii 3937, a null mutant of $r s s B$ (Ech160) was generated. The promoter activities of $h r p A$ and $h r p N$ were measured by flow cytometry. A decrease in $h r p A$ and $h r p N$ expression was observed in Ech160 compared with $D$. dadantii 3937 (Table 2). The expression of $h r p A$ was restored to the wild-type level when Ech160 was complemented with pMLrssB (Table 3 ). In addition, a decrease in $h r p L$ expression was observed in Ech160 when promoter activity was monitored by GFP promoter probe (Table 2). These results demonstrate that RssB acts as a positive regulator of

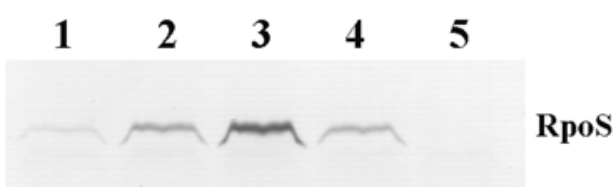

Fig. 4. Western blot with anti-RpoS ${ }_{\mathrm{Dd}}$ antibody. Cell extracts were prepared from cultures grown in minimal medium for $12 \mathrm{~h}$ at $28^{\circ} \mathrm{C}$. Lane 1, Dickeya dadantii 3937 (WT); lane 2, Ech157 (clpX mutant); lane 3, Ech156 (clpP mutant); lane 4, Ech160 (rssB mutant), lane 5, Ech159 (rpoS mutant).
T3SS. This also suggests that RssB may play a role as a direct recognition factor in ClpXP-dependent RpoS proteolysis in $D$. dadantii 3937.

We hypothesized that ClpXP may regulate T3SS through degradation of RpoS in $D$. dadantii 3937. To determine whether attenuated $h r p A$ expression in $\operatorname{clp} P, \operatorname{clpX}$, and $r s s B$ mutants was due to accumulation of RpoS, protein expression was analyzed by Western blot using anti-RpoS $S_{\mathrm{Dd}}$ antibodies and total cell extracts from Ech156 (clpP mutant), Ech157 (clpX mutant), and Ech160 (rssB mutant) grown in MM. Western blot analysis revealed that, compared with the wild type, RpoS accumulated to a higher level in Ech156, Ech157, and Ech160 (Fig. 4), suggesting that ClpXP and RssB are responsible for the degradation of RpoS. The diminished hrpA expression level in strains Ech156, Ech157, and Ech160 is likely due to increased amounts of RpoS. Together, the results above demonstrate that ClpXP regulates T3SS through degradation of RpoS in D. dadantii 3937.

\section{Effect of ClpXP, RssB, and RpoS}

on pectinolytic enzyme production and virulence.

In addition to their effect on the T3SS, ClpXP, RssB, and RpoS were evaluated for their role in Pel production in $D$. dadantii. Cultures of Ech156 (clpP mutant), Ech157 (clpX mutant), Ech158 (clpXP mutant), Ech159 (rpoS mutant), Ech160 (rss $B$ mutant), and the wild-type strain were tested for total Pel activity by a spectrophotometric assay. Compared with the wild-type strain, Ech156, Ech157, Ech158, and Ech160 showed a considerable reduction in Pel activity (Fig. 5). However, an increase in Pel activity was observed in Ech159 (Fig. 5). Because RsmA was reported to suppress the production of pectinolytic enzymes in $P$. carotovorum (Cui et al. 1995), the effect of ClpXP, RssB, and RpoS on Pel production in $D$. dadantii 3937 may be due to their effect on $r s m A$ expression (Fig. 1).

Both pectinolytic enzymes and the T3SS play major roles in the pathogenicity of D. dadantii (Collmer and Keen 1986; Bauer et al. 1994; Roy et al. 1999; Herron et al. 2000; Yang et al. 2002; Kazemi-Pour et al. 2004). Ech156, Ech157, Ech158, Ech159, and Ech160 were evaluated for the ability to cause disease on Chinese cabbage. Compared with the wild-type strain, reduced maceration symptoms were observed in Ech156, Ech157, Ech158, and Ech160 (Fig. 6), which is likely due to the significant reduction in Pel production and T3SS expression

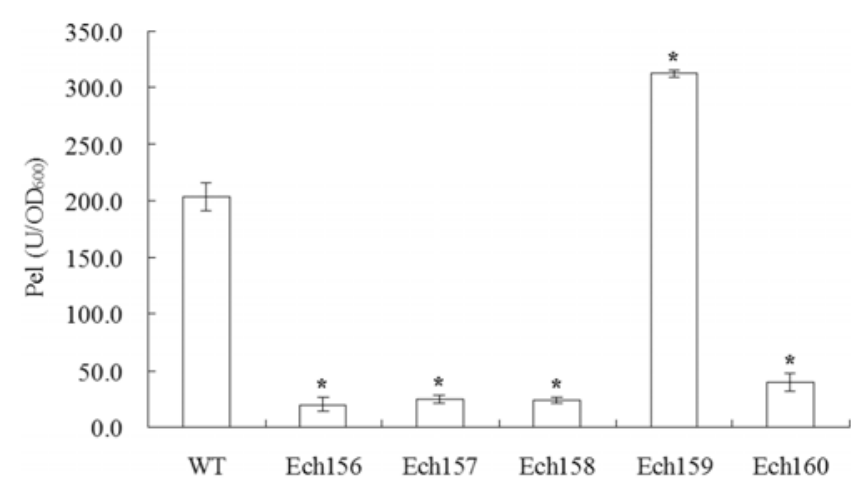

Fig. 5. Production of total pectate lyases (Pel) in Dickeya dadantii 3937 (WT), clpP mutant (Ech156), clpX mutant (Ech157), clpXP mutant (Ech158), rpoS mutant (Ech159), and rssB mutant (Ech160). Spectrophotometric quantification of Pel activity (unit per optical density at $600 \mathrm{~nm}$ ) was examined as described (Matsumoto et al. 2003). Values are a representative of two individual experiments. Three replicates were used in each experiment. Value is presented as the average of three replicates. Asterisks indicate statistically significant differences in Pel activity of the mutants compared with the wild type $(P<0.05$, Student's $t$ test). 
in these mutants. The rpoS mutant (Ech159) showed increased maceration ability (Fig. 6), probably because of the increase in Pel production and T3SS expression. During starvation and environmental stresses, the RssB activity is sequestered, which results in stabilization of the RpoS protein (Bougdour et al. 2006). An increase in the cellular levels of RpoS through RssB regulation suggests that $\mathrm{RpoS}$ may play a role in bacterial virulence in response to environmental conditions.

In summary, the regulatory roles of ClpXP, RssB, and RpoS in D. dadantii 3937 are depicted in Figure 1. With the assistance of the recognition factor RssB, ClpXP degrades the sigma factor, RpoS. RpoS negatively regulates promoter activity of $h r p L$. This regulatory action could possibly be indirect through an unidentified transcriptional repressor of $h r p L$ which is upregulated by RpoS. In addition, RpoS positively regulates rsmA expression. RsmA promotes the degradation of $h r p L$ and pel mRNA and, ultimately, leads to a reduction in T3SS gene expression and Pel production. Furthermore, ClpXP, RssB, and RpoS play an essential role in the virulence of $D$. dadantii 3937 by regulating the components necessary for T3SS gene expression and Pel production.

\section{MATERIALS AND METHODS}

\section{Bacterial strains, plasmids, primers, and media.}

The bacterial strains and plasmids used in this study are listed in Table 1. D. dadantii 3937 and mutant strains were stored at $-80^{\circ} \mathrm{C}$ in $15 \%$ glycerol. D. dadantii strains were grown in Luria-Bertani (LB) medium (1\% tryptone, $0.5 \%$ yeast extract, and $1 \% \mathrm{NaCl})$, MG medium (1\% mannitol, $0.2 \%$ glutamic acid, $0.05 \%$ potassium phosphate monobasic, $0.02 \% \mathrm{NaCl}$, and $0.02 \% \mathrm{MgSO}_{4}$ ) or low-nutrient T3SSinducing $\mathrm{MM}$ at $28^{\circ} \mathrm{C}$ (Yang et al. 2007). E. coli strains were grown in $\mathrm{LB}$ at $37^{\circ} \mathrm{C}$. Antibiotics were added at the following concentrations when required: ampicillin (100 $\mu \mathrm{g} / \mathrm{ml})$, kanamycin $(50 \mu \mathrm{g} / \mathrm{ml})$, gentamicin $(10 \mu \mathrm{g} / \mathrm{ml})$, and spectinomycin $(50 \mu \mathrm{g} / \mathrm{ml})$. Primers used for PCR in this report are listed in Supplementary Table 1.

\section{Construction of plasmids and mutants.}

The $\operatorname{clpP}$ (Ech156), clpX (Ech157), clpXP (Ech158), rpoS (Ech159), rssB (Ech160), and rpoE (Ech161) deletion mutants were constructed by marker exchange mutagenesis (Yang et al. 2002). Briefly, two fragments flanking each target gene were obtained. A kanamycin cassette, amplified from pKD4 (Table 1), was ligated with these two fragments and then cloned into BamHI and XhoI sites in pWM91. This construct was transferred into D. dadantii 3937 by conjugation using E. coli S17-1. To select strains with chromosomal deletions, transconjugants with kanamycin and ampicillin resistance were plated on MG containing 5\% sucrose and kanamycin. Colonies having sucrose and kanamycin resistance and ampicillin sensitivity were isolated and confirmed by PCR using outside primers. To construct plasmids for complementation, the coding regions of target genes were amplified and were cloned into low-copy number plasmids pML122 or pML123 (Table 1). All of the constructs and mutants described above were verified by PCR and DNA sequencing.

\section{Flow cytometry analysis.}

To screen for regulators that alter the expression of $h r p A$, the miniHimar RB1 transposon (Bouhenni et al. 2005) was used to construct a mutant library of $D$. dadantii 3937 . A reporter plasmid carrying a transcriptional fusion of the $h r p A$ promoter controlling expression of $g f p$ (phrpA) was mobilized into each transposon mutant and the hrpA promoter activity was monitored by flow cytometry as previously described (BD Biosciences, San Jose, CA, U.S.A.) (Peng et al. 2006). One of these mutants showed reduced expression of $h r p A$, and the site of the transposon insertion was identified as $c l p P$.

Promoter- $g f p$ constructs phrpA, phrpN, pdspE, phrpS, and phrpL were used to monitor promoter activities in $D$. dadantii strains by flow cytometry. Briefly, the bacterial cells carrying the promoter-GFP transcriptional fusions were grown in LB at $28^{\circ} \mathrm{C}$ overnight and transferred to $\mathrm{MM}$ to induce T3SS gene expression. Samples were collected and diluted to the appropriate concentration with $1 \times$ phosphate-buffered saline. The promoter activity was analyzed by measuring GFP intensity using flow cytometry.

\section{qRT-PCR analysis.}

qRT-PCR was used to measure transcript levels of target genes. Bacteria were cultured in MM for $12 \mathrm{~h}$. Cells were harvested and total RNA was isolated by using TRI reagent (Sigma-Aldrich, St. Louis) in combination with the RNeasy Mini kit (Qiagen Sciences, Germantown, MD, U.S.A.) and the TURBO DNA-free kit (Ambion, Austin, TX, U.S.A.) as described elsewhere (Peng et al. 2006; Li et al. 2009). cDNA was synthesized using the iScript cDNA synthesis kit (Bio-Rad, Hercules, CA, U.S.A.) according to the manufacturer's instructions. cDNA levels of target genes were quantified by qRTPCR using RealMaster SYBR ROX Mix (5 PRIME, Gaithersburg, MD, U.S.A.), with $r p l U$ as an endogenous control for data analysis (Mah et al. 2003; Kuchma et al. 2005).

\section{Northern blot analysis.}

Bacterial strains were grown in MM for $12 \mathrm{~h}$, and total RNA was isolated by using TRI reagent and treated with the TURBO DNA-free kit. RNA samples were analyzed by using biotin-labeled $h r p A, r s m B$, or $r s m A$ probes and a biotin detection system (BrightStar Psoralen-Biotin and Bright Star BioDetect; Ambion). 16S rRNA was used as an internal control.
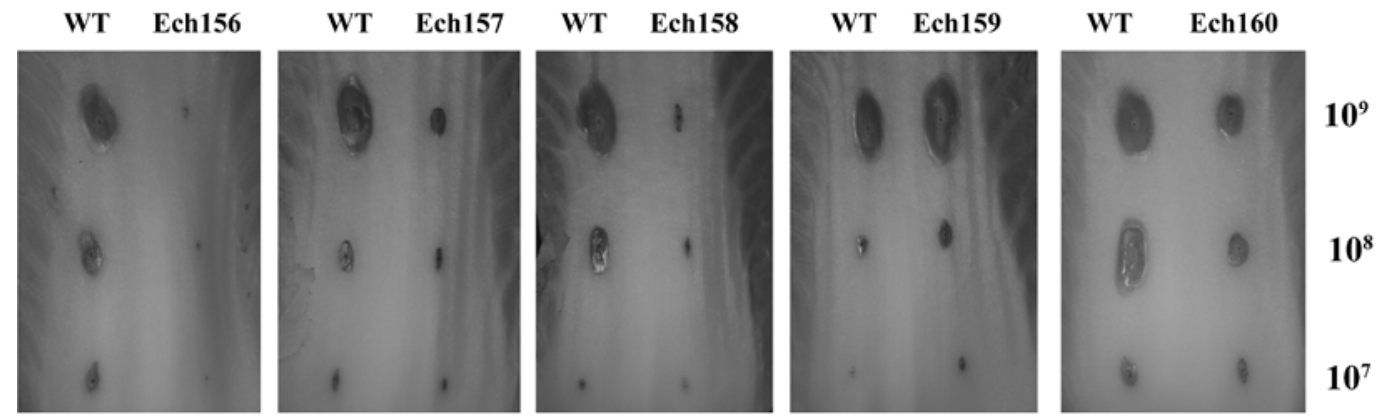

Fig. 6. Disease lesions caused by Dickeya dadantii 3937 (WT), clpP mutant (Ech156), clpX mutant (Ech157), clpXP mutant (Ech158), rpoS mutant (Ech159), and $r s s B$ mutant (Ech160) in leaves of Chinese cabbage. The leaves were inoculated at small wounds with a 10- $\mu$ l bacterial suspension at concentrations of $10^{7}, 10^{8}$, and $10^{9} \mathrm{CFU} / \mathrm{ml}$. Pictures were taken at $36 \mathrm{~h}$ after inoculation. 


\section{Western blot analysis.}

Bacteria were cultured in $\mathrm{MM}$ at $28^{\circ} \mathrm{C}$ for $12 \mathrm{~h}$. Cells were harvested and resuspended in bacterial protein extraction reagent (B-PER; Thermo Scientific, Rockford, IL, U.S.A.) supplemented with lysozyme $(100 \mathrm{ng} / \mathrm{ml})$. Samples were incubated for $30 \mathrm{~min}$ at room temperature and centrifuged to remove cell debris. The protein concentration was determined using a BioRad protein assay (Bio-Rad) and normalized to an equivalent concentration by adding B-PER. Proteins from total cell lysate were separated by sodium dodecyl sulfate polyacrylamide gel electrophoresis and then transferred to an Immobilion polyvinylidene diflouride transfer membrane (Millipore, Bedford, MA, U.S.A.) using a Trans-Blot SD Semi-Dry Transfer Cell (Bio-Rad). The blot was sequentially probed with a $1: 10,000$ dilution of anti-RpoS ${ }_{\text {Dd }}$ peptide antibody and a 1:500 dilution of an alkaline phosphatase-conjugated goat anti-rabbit immunoglobulin G secondary antibody (Millipore). Chromogenic detection was carried out with nitro-blue tetrazolium chloride and 5-bromo-4-chloro-3-indolyl phosphate $p$-toluine salt. To develop anti-RpoS $S_{\mathrm{Dd}}$ antibodies, a short-peptide fragment in the $\mathrm{RpoS}_{\mathrm{Dd}}$ protein sequence (ILQVQGLDIEELFRE) was designed, synthesized, conjugated to keyhole limpet hemocyanin, and purified by Proteintech Group, Inc. (Chicago). Rabbits were immunized against the short-peptide fragments and boosted four times after the primary immunization. The antibody value was tested further at different intervals after the primary immunization. Anti-RpoS $S_{\text {Dd }}$ peptide antibodies were affinity purified using the synthesized peptides that were used for immunization (Proteintech Group, Inc.).

\section{Enzyme activity assay.}

Spectrophotometric quantification of Pel activity was performed essentially as previously described (Matsumoto et al. 2003). Briefly, cells from overnight cultures grown in LB were subcultured in $\mathrm{MM}$ containing $1 \%$ polygalacturonic acid (PGA) at $28^{\circ} \mathrm{C}$ for $20 \mathrm{~h}$. The optical density of cell suspensions was adjusted to 1.0 at $600 \mathrm{~nm}\left(\mathrm{OD}_{600}\right)$ by adding $\mathrm{MM}$ with 1\% PGA. For total Pel activity, $1 \mathrm{ml}$ of the culture was sonicated two times for $20 \mathrm{~s}$ (Virsonic 600; VirTis, Gardiner, NY, U.S.A.) on ice and then centrifuged at $15,000 \mathrm{rpm}$ for 2 min to remove cell debris, and the resultant supernatant was used for the assay. In all, $10 \mu \mathrm{l}$ of sample solution was added to $990 \mu \mathrm{l}$ of the reaction buffer $(0.05 \%$ PGA, $0.1 \mathrm{M}$ Tris-HCl [pH 8.5], and $0.1 \mathrm{mM} \mathrm{CaCl}_{2}$, prewarmed to $30^{\circ} \mathrm{C}$ ) and thoroughly mixed. The $\mathrm{OD}_{230}$ was monitored for $3 \mathrm{~min}$. One unit of Pel activity was equivalent to an increase of $1 \times 10^{-3} \mathrm{OD}_{230}$ in $1 \mathrm{~min}$.

\section{Leaf maceration assay.}

The $D$. dadantii 3937 and mutant strains were grown in LB at $28^{\circ} \mathrm{C}$ overnight. Cells were harvested and resuspended in 50 $\mathrm{mM}$ phosphate buffer ( $\mathrm{pH} 7.4$ ) and adjusted to $10^{7}, 10^{8}$, and $10^{9} \mathrm{CFU} / \mathrm{ml}$. Chinese cabbage leaves were used for the local maceration assay. For each Chinese cabbage leaf, the wild type and mutant strain were inoculated in parallel by addition of 10 $\mu \mathrm{l}$ of the appropriate culture. Leaves were observed for maceration symptoms $36 \mathrm{~h}$ after inoculation. Maceration assays were performed in triplicate.

\section{ACKNOWLEDGMENTS}

This work is dedicated to Noel T. Keen. We thank N. Perna of the University of Wisconsin Madison for providing access to the annotated $D$. dadantii genome sequences. This project is supported by grants from the National Science Foundation (award no. EF-0332163), the Research Growth Initiative of the University of Wisconsin-Milwaukee, and the State Scholarship Fund of the China Scholarship Council awarded to Y. Li.

\section{LITERATURE CITED}

Ades, S. E., Connolly, L. E., Alba, B. M., and Gross, C. A. 1999. The Escherichia coli sigma(E)-dependent extracytoplasmic stress response is controlled by the regulated proteolysis of an anti-sigma factor. Genes Dev. 13:2449-2461.

Antunez-Lamas, M., Cabrera-Ordonez, E., Lopez-Solanilla, E., Raposo, R., Trelles-Salazar, O., Rodriguez-Moreno, A., and RodriguezPalenzuela, P. 2009. Role of motility and chemotaxis in the pathogenesis of Dickeya dadantii 3937 (ex Erwinia chrysanthemi 3937). Microbiology 155:434-442.

Bauer, D. W., Bogdanove, A. J., Beer, S. V., and Collmer, A. 1994. Erwinia chrysanthemi hrp genes and their involvement in soft rot pathogenesis and elicitation of the hypersensitive response. Mol. Plant-Microbe Interact. 7:573-581.

Bougdour, A., Wickner, S., and Gottesman, S. 2006. Modulating RssB activity: IraP, a novel regulator of sigma(S) stability in Escherichia coli. Genes Dev. 20:884-897.

Bouhenni, R., Gehrke, A., and Saffarini, D. 2005. Identification of genes involved in cytochrome c biogenesis in Shewanella oneidensis, using a modified mariner transposon. Appl. Environ. Microbiol. 71:4935-4937.

Butler, S. M., Festa, R. A., Pearce, M. J., and Darwin, K. H. 2006. Selfcompartmentalized bacterial proteases and pathogenesis. Mol. Microbiol. 60:553-562.

Chatterjee, A., Cui, Y., Liu, Y., Dumenyo, C. K., and Chatterjee, A. K. 1995. Inactivation of rsmA leads to overproduction of extracellular pectinases, cellulases, and proteases in Erwinia carotovora subsp. carotovora in the absence of the starvation/cell density-sensing signal, $\mathrm{N}$-(3oxohexanoyl)-L-homoserine lactone. Appl. Environ. Microbiol. 61:1959-1967.

Chatterjee, A., Cui, Y., and Chatterjee, A. K. 2002. Regulation of Erwinia carotovora $h r p L_{(E c))}\left(\right.$ sigma- $\left.L_{(E c)}\right)$, which encodes an extracytoplasmic function subfamily of sigma factor required for expression of the HRP regulon. Mol. Plant-Microbe Interact. 15:971-980.

Collmer, A., and Keen, N. T. 1986. The role of pectic enzymes in plant pathogenesis. Annu. Rev. Phytopathol. 24:383-409.

Cui, Y., Chatterjee, A., Liu, Y., Dumenyo, C. K., and Chatterjee, A. K. 1995. Identification of a global repressor gene, rsmA, of Erwinia carotovora subsp. carotovora that controls extracellular enzymes, $\mathrm{N}$-(3-oxohexanoyl)-L-homoserine lactone, and pathogenicity in soft-rotting $\mathrm{Er}$ winia spp. J. Bacteriol. 177:5108-5115.

Datsenko, K. A., and Wanner, B. L. 2000. One-step inactivation of chromosomal genes in Escherichia coli K-12 using PCR products. Proc. Natl. Acad. Sci. U.S.A. 97:6640-6645.

Dougan, D. A., Mogk, A., Zeth, K., Turgay, K., and Bukau, B. 2002. $\mathrm{AAA}+$ proteins and substrate recognition, it all depends on their partner in crime. FEBS (Fed. Eur. Biochem. Soc.) Lett. 529:6-10.

Ehrmann, M., and Clausen, T. 2004. Proteolysis as a regulatory mechanism. Annu. Rev. Genet. 38:709-724.

Franza, T., Sauvage, C., and Expert, D. 1999. Iron regulation and pathogenicity in Erwinia chrysanthemi 3937: role of the Fur repressor protein. Mol. Plant-Microbe Interact. 12:119-128.

Gottesman, S. 1996. Proteases and their targets in Escherichia coli. Annu. Rev. Genet. 30:465-506.

Gottesman, S. 2003. Proteolysis in bacterial regulatory circuits. Annu. Rev. Cell Dev. Biol. 19:565-587.

Hayden, J. D., and Ades, S. E. 2008. The extracytoplasmic stress factor, sigmaE, is required to maintain cell envelope integrity in Escherichia coli. PLoS ONE 3:e1573.

He, S. Y., Nomura, K., and Whittam, T. S. 2004. Type III protein secretion mechanism in mammalian and plant pathogens. Biochim. Biophys. Acta 1694:181-206.

Hengge-Aronis, R. 2002. Signal transduction and regulatory mechanisms involved in control of the sigma(S) (RpoS) subunit of RNA polymerase. Microbiol. Mol. Biol. Rev. 66:373-395.

Herron, S. R., Benen, J. A., Scavetta, R. D., Visser, J., and Jurnak, F. 2000. Structure and function of pectic enzymes: virulence factors of plant pathogens. Proc. Natl. Acad. Sci. U.S.A. 97:8762-8769.

Hueck, C. J. 1998. Type III protein secretion systems in bacterial pathogens of animals and plants. Microbiol. Mol. Biol. Rev. 62:379-433.

Iyoda, S., and Watanabe, H. 2005. ClpXP protease controls expression of the type III protein secretion system through regulation of RpoS and GrlR levels in enterohemorrhagic Escherichia coli. J. Bacteriol. 187:4086-4094.

Jenal, U., and Hengge-Aronis, R. 2003. Regulation by proteolysis in bacterial cells. Curr. Opin. Microbiol. 6:163-172.

Kazemi-Pour, N., Condemine, G., and Hugouvieux-Cotte-Pattat, N. 2004. The secretome of the plant pathogenic bacterium Erwinia chrysanthemi. Proteomics 4:3177-3186.

Kuchma, S. L., Connolly, J. P., and O’Toole, G. A. 2005. A three-compo- 
nent regulatory system regulates biofilm maturation and type III secretion in Pseudomonas aeruginosa. J. Bacteriol. 187:1441-1454.

Labes, M., Puhler, A., and Simon, R. 1990. A new family of RSF1010derived expression and lac-fusion broad-host-range vectors for gramnegative bacteria. Gene 89:37-46.

Lange, R., and Henggearonis, R. 1994. The cellular concentration of the Sigma(S) subunit of RNA polymerase in Escherichia coli is controlled at the levels of transcription translation, and protein stability. Genes Dev. 8:1600-1612.

Li, Y., Peng, Q., Selimi, D., Wang, Q., Charkowski, A.O., Chen, X., and Yang, C. H. 2009. The plant phenolic compound p-coumaric acid represses gene expression in the Dickeya dadantii type III secretion system. Appl. Environ. Microbiol. 75:1223-1228.

Liu, Y., Cui, Y. Y., Mukherjee, A., and Chatterjee, A. K. 1998. Characterization of a novel RNA regulator of Erwinia carotovora ssp. carotovora that controls production of extracellular enzymes and secondary metabolites. Mol. Microbiol. 29:219-234.

Loewen, P. C., Hu, B., Strutinsky, J., and Sparling, R. 1998. Regulation in the rpoS regulon of Escherichia coli. Can. J. Microbiol. 44:707-717.

Ma, B., Hibbing, M. E., Kim, H. S., Reedy, R. M., Yedidia, I., Breuer, J., Glasner, J. D., Perna, N. T., Kelman, A., and Charkowski, A. O. 2007. Host range and molecular phylogenies of the soft rot enterobacterial genera Pectobacterium and Dickeya. Phytopathology 97:1150-1163.

Matsumoto, H., Muroi, H., Umehara, M., Yoshitake, Y., and Tsuyumu, S. 2003. Peh production, flagellum synthesis, and virulence reduced in Erwinia carotovora subsp. carotovora by mutation in a homologue of cytR. Mol. Plant-Microbe Interact. 16:389-397.

McMeechan, A., Roberts, M., Cogan, T. A., Jorgensen, F., Stevenson, A., Lewis, C., Rowley, G., and Humphrey, T. J. 2007. Role of the alternative sigma factors sigmaE and sigmaS in survival of Salmonella enterica serovar Typhimurium during starvation, refrigeration and osmotic shock. Microbiology 153:263-269.

Metcalf, W. W., Jiang, W., Daniels, L. L., Kim, S. K., Haldimann, A., and Wanner, B. L. 1996. Conditionally replicative and conjugative plasmids carrying lac $Z \alpha$ for cloning, mutagenesis, and allele replacement in bacteria. Plasmid 35:1-13.

Miller, W. G., Leveau, J. H. J., and Lindow, S. E. 2000. Improved $g f p$ and inaZ broad-host-range promoter-probe vectors. Mol. Plant-Microbe Interact. 13:1243-1250.

Missiakas, D., Mayer, M. P., Lemaire, M., Georgopoulos, C., and Raina, S. 1997. Modulation of the Escherichia coli sigmaE (RpoE) heat-shock transcription-factor activity by the RseA, RseB and RseC proteins. Mol. Microbiol. 24:355-371.

Mota, L. J., Sorg, I., and Cornelis, G. R. 2005. Type III secretion: the bacteria-eukaryotic cell express. FEMS (Fed. Eur. Microbiol. Soc.) Microbiol. Lett. 252:1-10.

Mukherjee, A., Cui, Y., Ma, W., Liu, Y., Ishihama, A., Eisenstark, A., and Chatterjee, A. K. 1998. RpoS (sigma-S) controls expression of rsmA, a global regulator of secondary metabolites, harpin, and extracellular proteins in Erwinia carotovora. J. Bacteriol. 180:3629-3634.

Ortega, J., Lee, H. S., Maurizi, M. R., and Steven, A. C. 2004. ClpA and ClpX ATPases bind simultaneously to opposite ends of ClpP peptidase to form active hybrid complexes. J. Struct. Biol. 146:217-226.

Peng, Q., Yang, S., Charkowski, A. O., Yap, M. N., Steeber, D. A., Keen, N. T., and Yang, C. H. 2006. Population behavior analysis of $d s p E$ and
pelD regulation in Erwinia chrysanthemi 3937. Mol. Plant-Microbe Interact. 19:451-457.

Porankiewicz, J., Wang, J., and Clarke, A. K. 1999. New insights into the ATP-dependent Clp protease: Escherichia coli and beyond. Mol. Microbiol. 32:449-458

Roy, C., Kester, H., Visser, J., Shevchik, V., Hugouvieux-Cotte-Pattat, N. Robert-Baudouy, J., and Benen, J. 1999. Modes of action of five different endopectate lyases from Erwinia chrysanthemi 3937. J. Bacteriol. 181:3705-3709.

Sandercock, J. R., and Page, W. J. 2008. RpoS expression and the general stress response in Azotobacter vinelandii during carbon and nitrogen diauxic shifts. J. Bacteriol. 190:946-953.

Schweder, T., Lee, K. H., Lomovskaya, O., and Matin, A. 1996. Regulation of Escherichia coli starvation sigma factor (sigma S) by ClpXP protease. J. Bacteriol. 178:470-476.

Tang, X. Y., Xiao, Y. M., and Zhou, J. M. 2006. Regulation of the type III secretion system in phytopathogenic bacteria. Mol. Plant-Microbe Interact. 19:1159-1166.

Tomoyasu, T., Takaya, A., Handa, Y., Karata, K., and Yamamoto, T. 2005. ClpXP controls the expression of LEE genes in enterohaemorrhagic Es cherichia coli. FEMS (Fed. Eur. Microbiol. Soc.) Microbiol. Lett. 253:59-66.

Wei, Z. M., and Beer, S. V. 1995. hrpL activates Erwinia amylovora hrp gene transcription and is a member of the ECF subfamily of sigma factors. J. Bacteriol. 177:6201-6210.

Yang, C. H., Gavilanes-Ruiz, M., Okinaka, Y., Vedel, R., Berthuy, I., Boccara, M., Chen, J. W., Perna, N. T., and Keen, N. T. 2002. hrp genes of Erwinia chrysanthemi 3937 are important virulence factors. Mol. Plant-Microbe Interact. 15:472-480.

Yang, S., Zhang, Q., Guo, J., Charkowski, A.O., Glick, B. R., Ibekwe, A. M., Cooksey, D. A., and Yang, C. H. 2007. Global effect of indole-3acetic acid biosynthesis on multiple virulence factors of Erwinia chrysanthemi 3937. Appl. Environ. Microbiol. 73:1079-1088.

Yang, S., Peng, Q., San Francisco, M., Wang, Y., Zeng, Q., and Yang, C. H. 2008a. Type III secretion system genes of Dickeya dadantii 3937 are induced by plant phenolic acids. PLoS One 3:e2973.

Yang, S., Peng, Q., Zhang, Q., Yi, X., Choi, C. J., Reedy, R. M., Charkowski, A. O., and Yang, C. H. 2008b. Dynamic regulation of GacA in type III secretion, pectinase gene expression, pellicle formation, and pathogenicity of Dickeya dadantii (Erwinia chrysanthemi 3937). Mol. Plant-Microbe Interact. 21:133-142.

Yap, M. N., Yang, C. H., Barak, J. D., Jahn, C. E., and Charkowski, A. O. 2005. The Erwinia chrysanthemi type III secretion system is required for multicellular behavior. J. Bacteriol. 187:639-648.

Yu, A. Y., and Houry, W. A. 2007. ClpP: a distinctive family of cylindrical energy-dependent serine proteases. FEBS (Fed. Eur. Biochem. Soc.) Lett. 581:3749-3757.

Zhou, Y., Gottesman, S., Hoskins, J. R., Maurizi, M. R., and Wickner, S. 2001. The RssB response regulator directly targets sigma(S) for degradation by ClpXP. Genes Dev. 15:627-637.

\section{AUTHOR-RECOMMENDED INTERNET RESOURCE}

ASAP database and web interface: asap.ahabs.wisc.edu/asap/ASAP1.htm 